\title{
Implementation of Simulation Program for Modeling the Effective Resistivity of Nanometer Scale Film and Line Interconnects
}

\author{
A. Emre Yarimbiyik \\ Harry A. Schafft \\ Richard A. Allen \\ Mona E. Zaghloul \\ David L. Blackburn
}


NISTIR 7234

\title{
Implementation of Simulation Program for Modeling the Effective Resistivity of Nanometer Scale Film and Line Interconnects
}

\author{
A. Emre Yarimbiyik \\ Mona E. Zaghloul \\ The Department of Electric and Computer Engineering, \\ The George Washington University \\ Washington, DC 20052 \\ Harry A. Schafft \\ Richard A. Allen \\ David L. Blackburn \\ Semiconductor Electronics Division \\ Electronics and Electrical Engineering Laboratory
}

February 2006

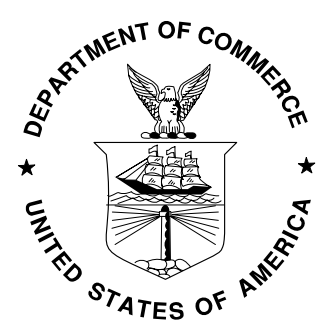

U.S. DEPARTMENT OF COMMERCE Carlos M. Gutierrez, Secretary TECHNOLOGY ADMINISTRATION Under Secretary of Commerce for Technology, William Jeffrey (acting as) NATIONAL INSTITUTE OF STANDARDS AND TECHNOLOGY William Jeffrey, Director 


\title{
Implementation of Simulation Program for Modeling the Effective Resistivity of Nanometer Scale Film and Line Interconnects
}

\author{
A. Emre Yarimbiyik ${ }^{1,2}$, Harry A. Schafft ${ }^{2}$, Richard A. Allen ${ }^{2}$, Mona E. Zaghloul ${ }^{1}$, \\ and David L. Blackburn ${ }^{2}$ \\ 1- The Department of Electric and Computer Engineering, \\ The George Washington University, Washington, DC 20052 \\ 2- Semiconductor Electronics Division, \\ National Institute of Standards and Technology, Gaithersburg, MD 20899
}

\begin{abstract}
A computer program that simulates the impact of the size effect on the effective resistivity of line and film conductors is described. Flowcharts and the program code are provided as appendices.

Introduction

A versatile program, with its flow chart and code, are provided to permit the reader to simulate how the effective resistivity of a metal conductor increases as the conductor cross sectional dimensions approach and become smaller than the bulk mean free path, $\lambda_{\max }$, of the conduction electrons (size effect). For copper $\lambda_{\max }=39 \mathrm{~nm}$ at room temperature. As integrated circuits (IC) are continuing to be designed with ever small conductor dimensions, the size effect on IC operating speed is a consideration of growing importance. (ITRS 2004 suggests dimensions smaller than $45 \mathrm{~nm}$ beyond 2009.) Hence, it is important to understand how grain size and the scattering characteristics of line and grain boundaries can increase the effective resistivity of lines and the R-C delays associated with the circuit wiring.

The program can model how scattering from surfaces and grain boundaries impact the effective resistivity of a film or line conductor when these scattering mechanisms act separately or simultaneously. The grain size, which may or may not be influenced by the dimensions of the conductor, is an important adjustable parameter in the program. The probability of an electron being scattered elastically from the surface can be made to be different for each surface of the conductor. This version of the program permits only one surface of a line to be different. Temperature effects are modeled by changing the value for the mean free path of the conduction electrons in the metal of interest. The application of this program was reported in another publication [1], where it was used to explore size effects in copper metallizations. While this program was designed for studying size effects in copper, it can be used equally well for other metallizations, such as aluminum and silver.
\end{abstract}




\section{Summary of the Simulation}

The code is developed for rectangular prism-shaped structures. Grain boundaries are assumed to be planes perpendicular to the sidewalls [3]. These planes are separated by a distance made equal to the estimated mean size of the grains in the metal. The simulation uses Sommerfeld's conductivity theory as expressed in eq. 1 to calculate the resistivity.

$$
\sigma=\frac{N_{f} e^{2} \tau}{m_{e f f}}
$$

where $N_{f}$ is the number of electrons with energy near the Fermi energy per unit volume, $e$ is the unit charge, $\tau$ is the relaxation time, and $m_{\text {eff }}$ is the effective mass of an electron in the lattice. To calculate the simulated resistivity, all that is needed is to calculate the average relaxation time for the electrons in the conductor. This is what the program is designed to do. Values for $N_{f}$ and the effective mass of the electron for a given metal exist in the literature and may be found in solid state physics books such as by Kittel [4].

To calculate the average relaxation time for the electrons in the conductor, the program simulates the movement of an electron in steps as it moves from one inelastic scattering event to the next. After each inelastic scattering event, the motion of the electron is modeled as moving at the Fermi speed, $v_{F}$, along a straight line that is directed in a randomly selected direction in the metal. If the electron is not scattered inelastically by a surface or grain boundary after it has traveled a distance equal to the bulk mean free path, $\lambda_{\max }$, it is assumed to be inelastically scattered. The relaxation time for the electron during this simulated step in its motion in the metal is set equal to its maximum value, $\tau_{\max }=\lambda_{\max } / \mathrm{v}_{\mathrm{F}}$. If the electron is inelastically scattered before this time, it is assigned a value for the relaxation time that is equal to the product of $\tau_{\max }$ and the fraction of $\lambda_{\max }$ that the electron has traveled during this step. The program calculates a value for the average of the relaxation times for each of $\mathrm{N}$ individual carriers (electrons) over $\mathrm{M}$ steps for a given geometry and grain size. Two parameters are used to characterize the scattering: $p$ is the probability of elastic (specular) scattering from a surface and $g$ is the probability of inelastic scattering by a grain boundary. Each of the $\mathrm{N}$ simulation electrons begins its first step by placing it in a randomly selected point in a grain and giving it a speed $v_{F}$ in a randomly selected direction.

\section{Setting the Parameters of the Program}

The following parameters are initialized in the start of the main function. 
MFP: Mean free path of electrons in metal. It is temperature dependent. This dependence is discussed in reference 1 .

MAXTREL: Maximum relaxation time for electrons in the pure, bulk form of the metal. The bulk resistivity of copper for different temperatures is listed in reference 5 . The user should calculate MAXTREL for copper using this information and equation 1.

pElastic: Surface scattering parameter, $p$, for three sides of the rectangular prism-shaped structure. Default value is 0.1. pElastic can take values between 0 and 1 .

pElasticFourthSide: $p$ value for the top side of the line. Default value is 0.1 . pElasticFourthSide can take values between 0 and 1 .

pGBSct: Grain boundary scattering parameter, $g$. Default value is 0.7 . pGBSct can take values between 0 and 1 .

Meff: Effective mass of electrons in the metal. It can be entered in terms of "M," the free electron mass, which is a constant in the program.

N_fermi: Number of electrons nearby the Fermi energy per volume. Note that this value is essentially temperature independent. For copper, it is equal to $8.47 \times 10^{28}$ [4].

N_simel: Number of simulation electrons. Default value is 500 .

N_step: Number of steps taken by each electron. Default value is 2000 .

N_simrange: Number of different geometries that are to be simulated in one run. Default value is 30 .

The parameters that define the dimensions are located in the "for" loop in the main function.

yvalue and zvalue: Width and height dimensions (note that all the lengths are in nanometers in the code) of the conductor line. For thin films, assigning 1000 times the "yvalue" to "zvalue" is a reasonably good approximation of a thin film. As the default, "yvalue" is $10 \mathrm{~nm}$ and is incremented by $10 \mathrm{~nm}$ in each loop. The number of loops is determined by "N_simrange."

grainSize: Average size of the grains. Grain size has crucial importance in taking the grain boundary scattering into account. Following typical usage reported in the literature, the default value for "grainSize" is the thickness of a film or the smaller cross sectional dimension of a line. However, to obtain the most accurate simulation results, use the value for the average size of the grains in the metallization determined from direct measurements. 


\section{REFERENCES}

[1] Yarimbiyik, A.E., Schafft, H.A., Allen, R.A., Zaghloul, M.E., Blackburn, D.L. Modeling and Simulation of Resistivity of Nanometer Scale Copper, In press Microelectronics Reliability 2006.

[2] Barnat, E.V., Nagakura, D., Wang, P-1, Lu, T-M. Real Time Resistivity Measurements During Sputter Deposition of Ultrathin Copper Films, JAP 2002;91:3:1667-72.

[3] Mayadas, A.F., Shatzkes, M. Electrical Resistivity Model for Polycrystalline Films: the Case of Arbitrary Reflection at External Surfaces, Phys. Rev. B 1970;1:4:1382-9.

[4] Kittel, C., Introduction to Solid State Physics. New York; John Wiley \& Sons, Inc., 1996; p. 150.

[5] Schuster, C.E., Vangel, M.G., Schafft, H.A. Improved Estimation of the Resistivity of Pure Copper and Electrical Determination of Thin Copper Film Dimensions, Microelectronics Reliability, 2001;41:239-52.

\section{APPENDIX 1. Program Flowcharts}

The following figures constitute the flowcharts of the Power Point file: FC_Cu.ppt.

Figure 1: Flowchart of the main routine.

Figure 2: Flowchart of the "Simulate Electron" function and a part of the main routine.

Figure 3: Flowchart of the elastic scattering routine.

Figure 4: Flowchart of the grain boundary check routine.

\section{APPENDIX 2. Program Code}

The code is given in a .java file, titled Copper.java. 


\section{Appendix 1 - Figure 1: Flowchart of the main routine}

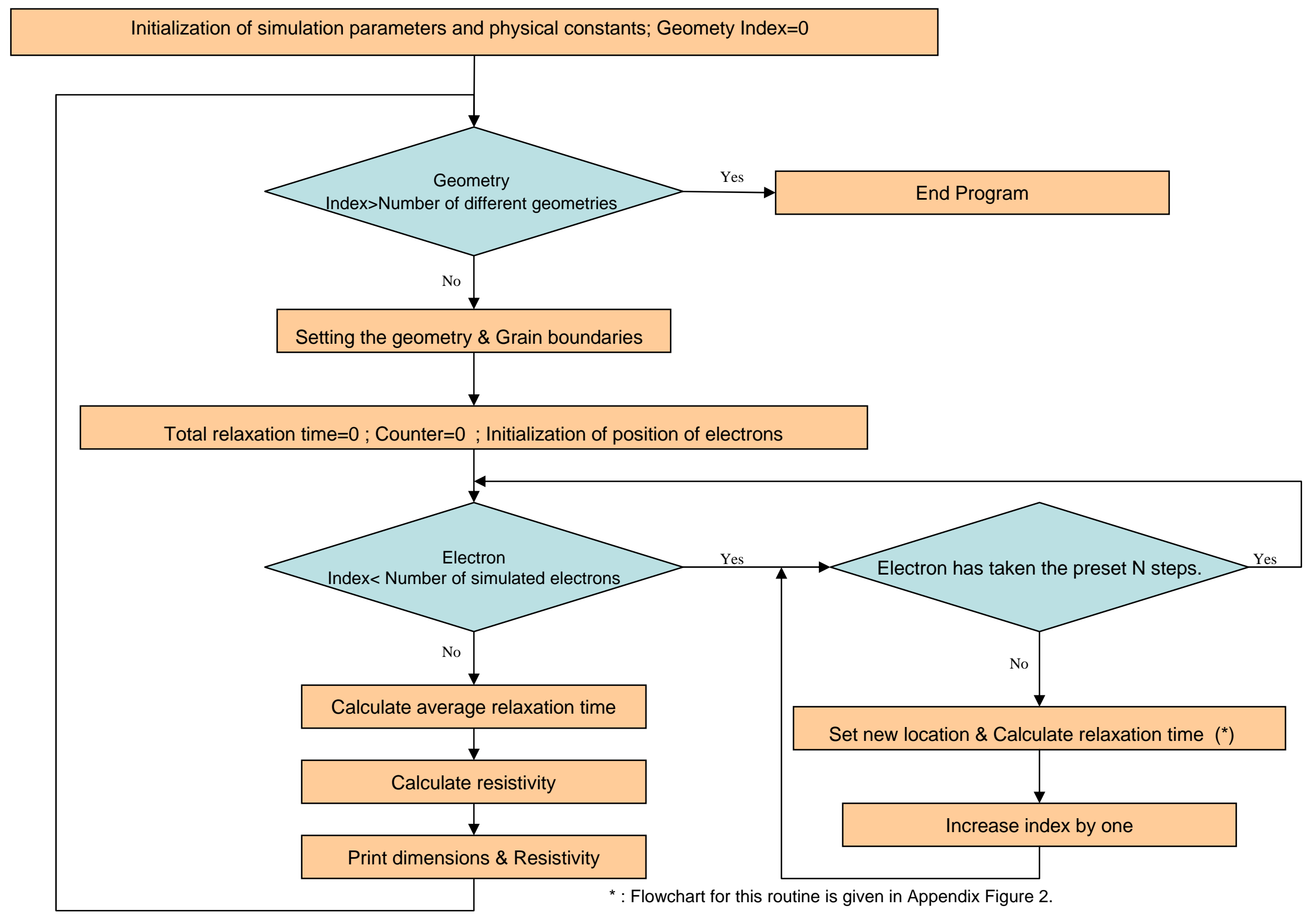


Find trajectory-surface boundary intersection point

$$
7
$$

Compute time until the surface boundary ( = tEL1) $\checkmark$

Find the position after elastic scattering

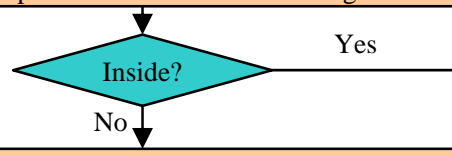

Find trajectory-surface boundary intersection point

$\checkmark$ Yes

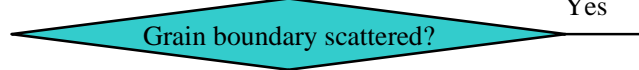

No $\frac{7}{2}$

Compute time until the surface boundary ( = tEL2 )

$$
7
$$

Inelastic scattering?

$$
\text { Not }
$$

Find the position after elastic scattering

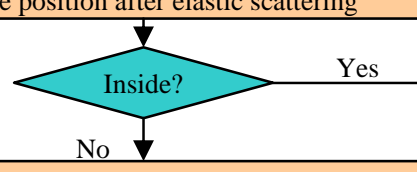

No $\downarrow$

Find trajectory-surface boundary intersection point

$$
\downarrow
$$

Grain boundary scattered?

$$
\text { Not }
$$

Compute time until the surface boundary ( = tEL3 )

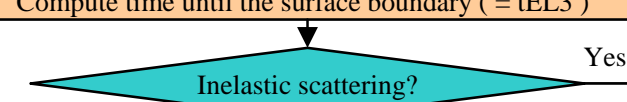

Inelastic scattering? No 7

Find the position after elastic scattering

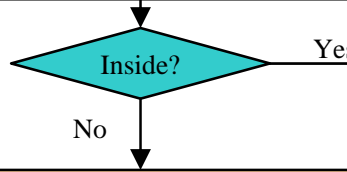

Yes

Find trajectory-surface boundary intersection point
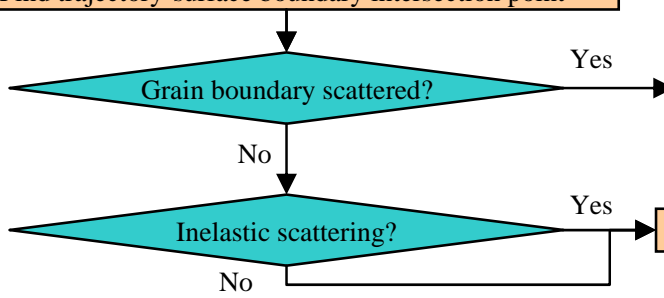

Appendix 1 - Figure 3: Flowchart of the elastic scattering routine

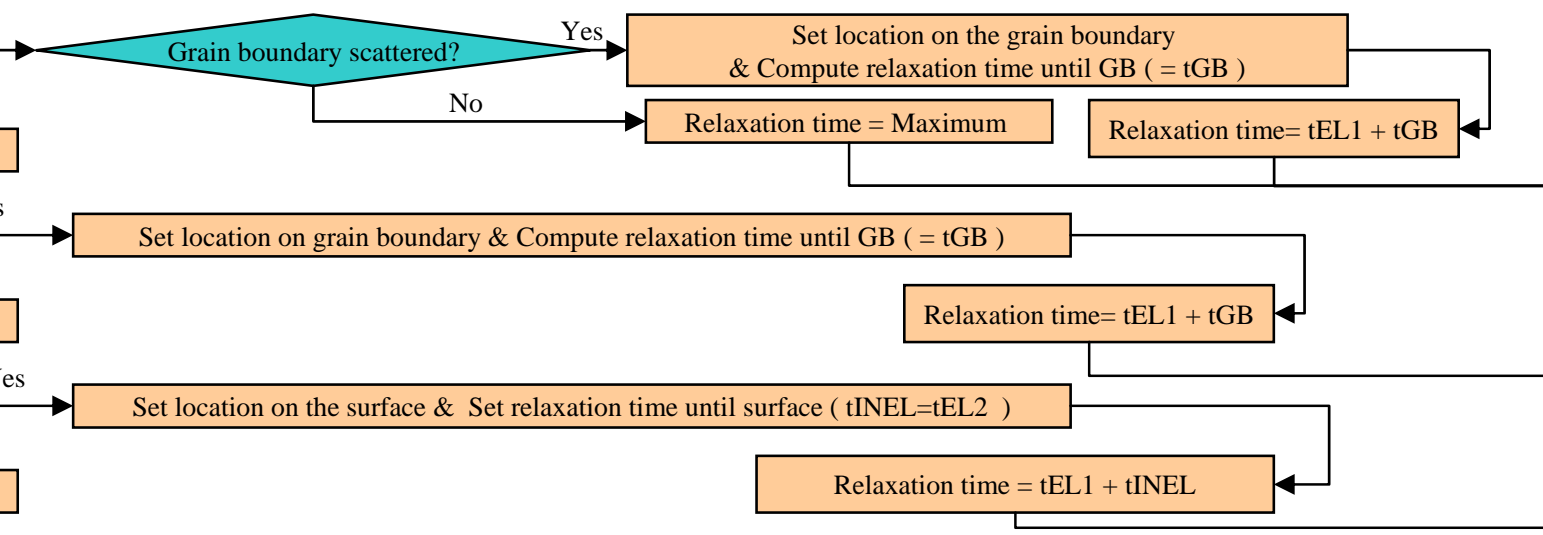

Set location on the grain boundary \& Compute relaxation time until GB ( = tGB )

Relaxation time $=$ Maximum

Relaxation time $=\mathrm{tEL} 1+\mathrm{tEL} 2+\mathrm{tGB}$

Set location on grain boundary \& Compute relaxation time until GB ( = tGB )

Relaxation time $=\mathrm{tEL} 1+\mathrm{tEL} 2+\mathrm{tGB}$

Set location on the surface $\&$ Set relaxation time until surface ( $t$ INEL=tEL3 )

Relaxation time $=\mathrm{tEL} 1+\mathrm{tEL} 2+\mathrm{tINEL}$

Yes Set location on the grain boundary \& Compute relaxation time until GB ( = tGB )

Relaxation time $=$ Maximum

Relaxation time $=\mathrm{tEL} 1+\mathrm{tEL} 2+\mathrm{tEL} 3+\mathrm{tGB}$

Set location on grain boundary \& Compute relaxation time until GB ( = tGB )

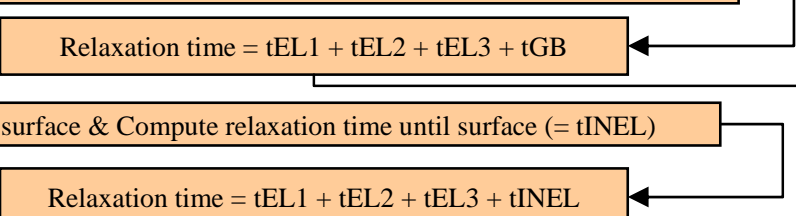




\section{Appendix 1 - Figure 4: Flowchart of the grain boundary check routine}

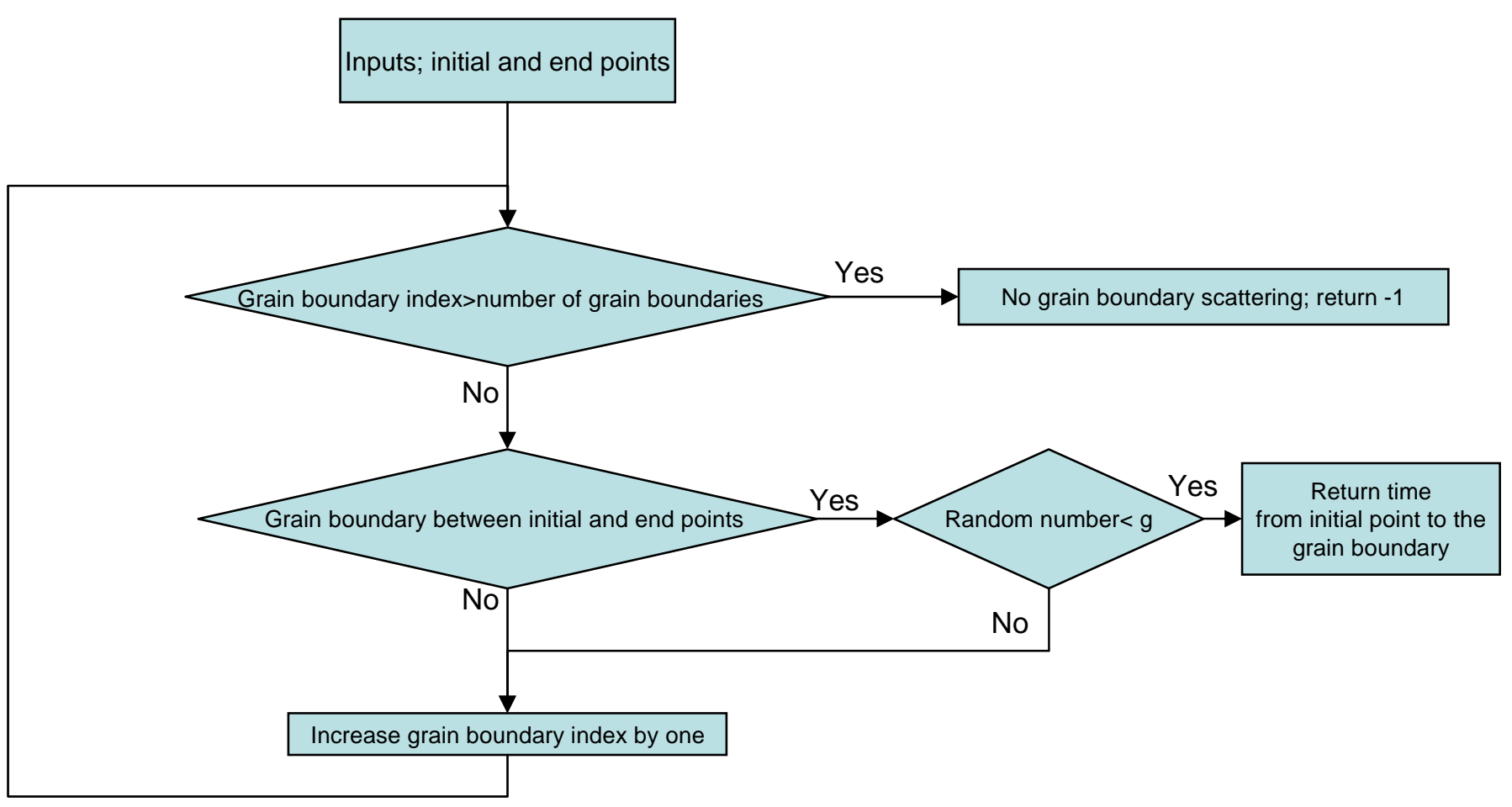

The locations of the grain boundaries are stored in an array in the simulation. When the function that checks for the grain boundary scattering is called, the elements of the array are checked to be between the $x$ coordinates of the initial and end points. If this condition is satisfied and the generated random number is bigger than the $g$ parameter, then the electron is scattered from the grain boundary and the loop breaks. If this double check yields a positive result for none of the array elements, then there is no grain boundary scattering. The "grain boundary scattering check" function returns -1 for no grain boundary scattering. If there is grain boundary scattering, the electron's position is set to the location that the electron's trajectory intersects with the grain boundary. In this case the return value is the time that passes for the electron to travel from its initial position to the grain boundary 


\section{APPENDIX 2 - JAVA Source Code}

\section{This code was written using the jGRASP editor and has successfully been compiled using the Java J2SE $v 1.4 .2$ - 08 SDK source code. A soft copy of the code is available from the authors.}

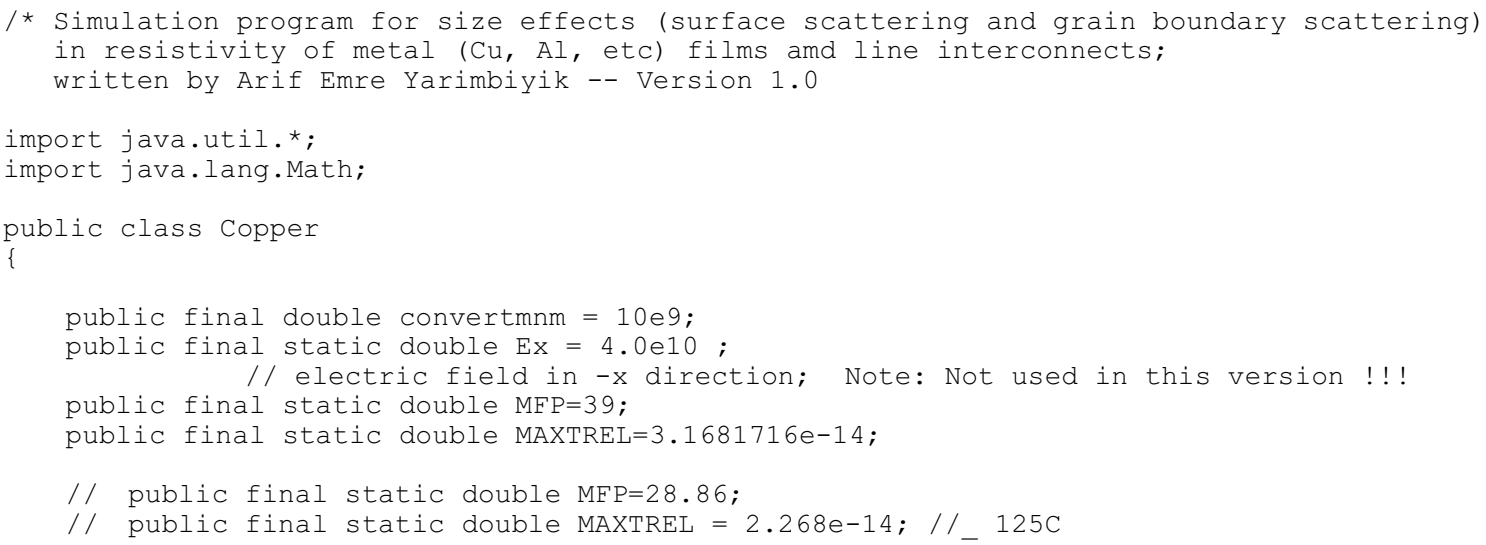




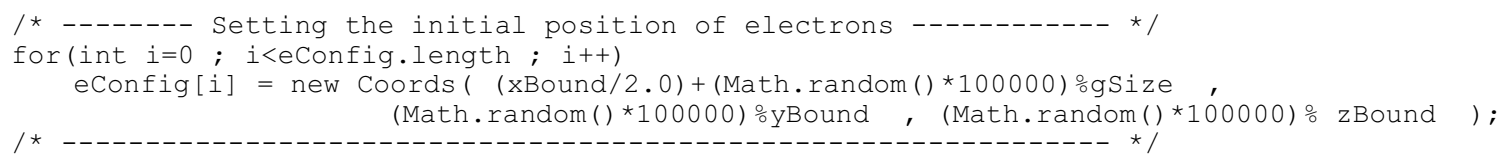




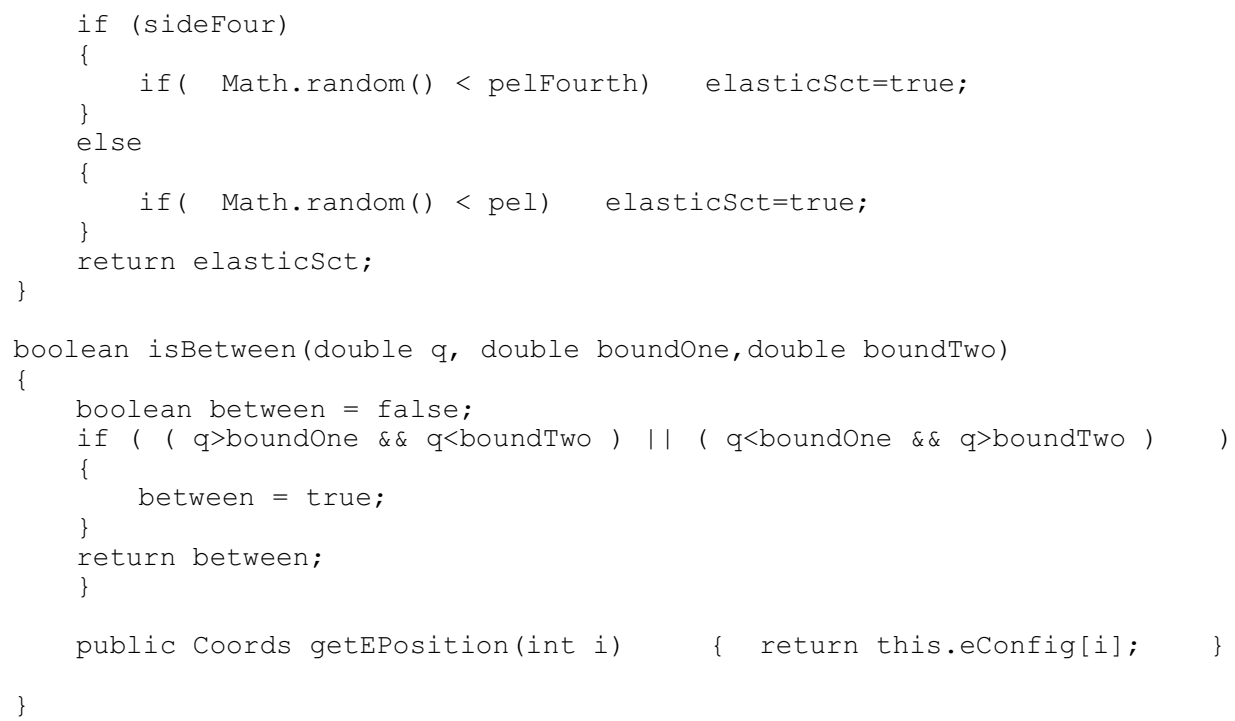

1111111111111111111111111111111111111111111111111111111111111111111111111111111

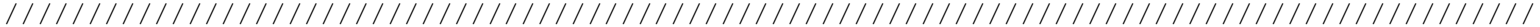

class Coords

\{

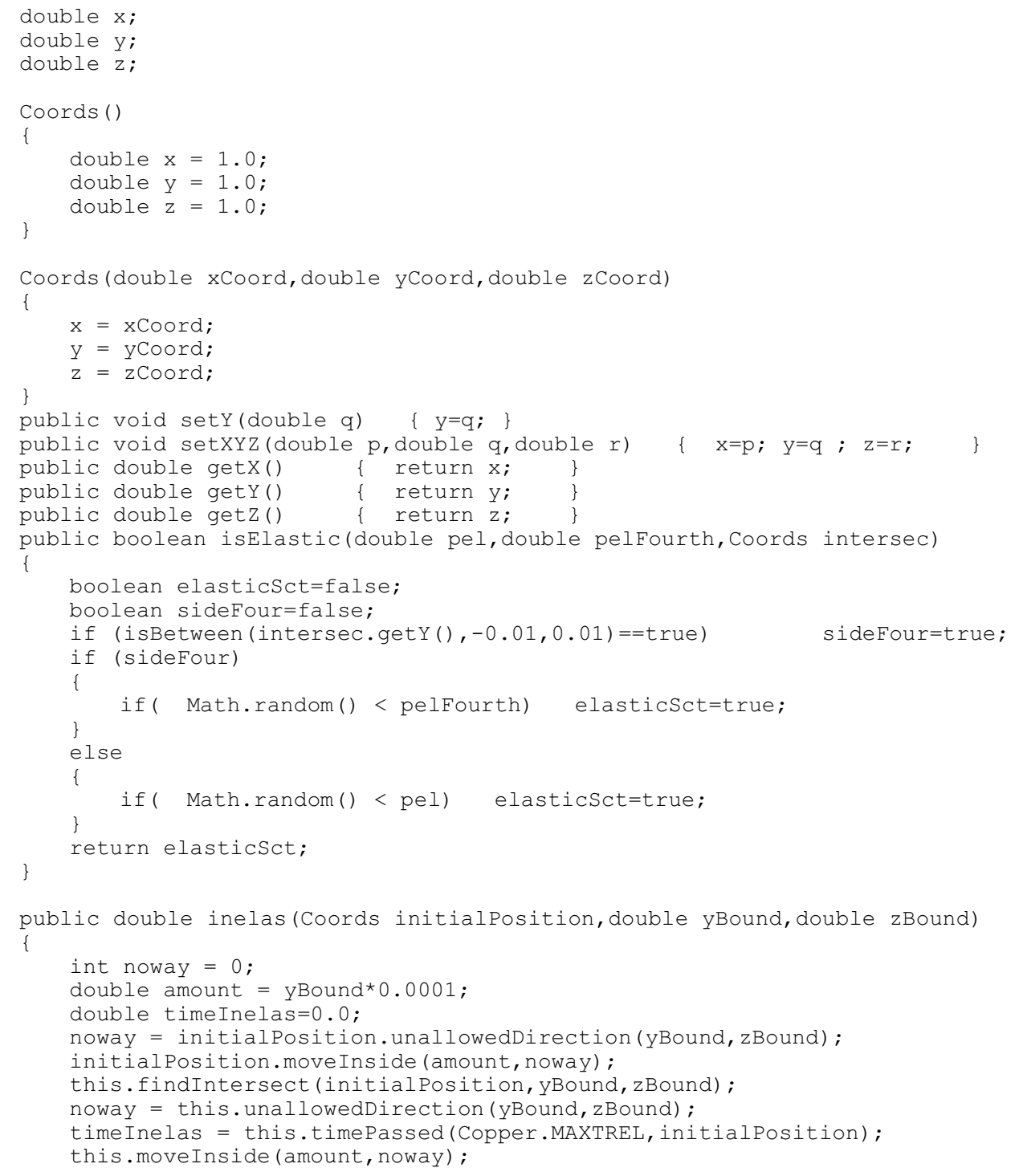


public double elas(Coords initialPosition, double xBound, double yBound, double zBound, double probElas, double probElasFourth, Coords [] grainB, double probGBS)

double timeElas=0.0, timeInelas=0.0;

double timeGBSct $=0.0$;

int noway;

Coords symmetric $=$ new Coords (this.getX(), this.getY(), this.getz());

this.findIntersect (initialPosition, yBound, zBound);

double t_elasfirst = this.timePassed(Copper.MAXTREL, initialPosition);

double $t$ elassecond $=0.0$;

initialPōsition.setXYZ(this.getX(), this.gety(), this.getz());

noway = initialPosition.unallowedDirection (yBound, zBound);

initialPosition.moveInside (0.00001, noway);

this.findElastic (symmetric, yBound, zBound);

boolean inside = this. checkifin (yBound, zBound);

if (inside)

\{

timeGBSct=this.gbSCT (initialPosition, grainB,probGBS);

if ( $t$ imeGBSct $==-1$. 0 )

\{

timeElas = Copper.MAXTREL ;

\}

timeInelas $=0.0$;

else

\{

timeInelas=timeGBSct;

timeElas=t_elasfirst;

else

\{

Coords newTempSurfacePosition = new Coords(this.getX(), this.getY(), this.getz());

newTempSurfacePosition. findIntersect (initialPosition, yBound, zBound) ;

timeGBSct=newTempSurfacePosition.gbSCT (initialPosition,grainB,probGBS) ;

if $(\mathrm{t}$ imeGBSCt $==-1$ )

\{

if (isElastic (probElas, probElasFourth, newTempSurfacePosition)==false)

timeInelas = this.inelas (initialPosition, yBound, zBound);

timeElas=t elasfirst

\}

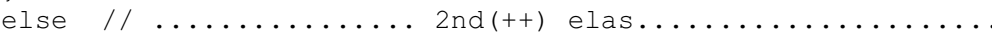

Coords symmetricTwo = new Coords(this.getX(), this.gety(), this.getz()) ; this.setXYZ( newTempSurfacePosition.getX(), newTempSurfacePosition.gety(), newTempsurfacePosition.getz ());

noway = this.unallowedDirection (yBound, zBound)

this.moveInside (0.00001, noway);

t_elassecond = this.timePassed (Copper.MAXTREL, initialPosition);

iñtialPosition.setXYZ(this.getX(), this.getY(), this.getz());

this.findElastic (symmetricTwo, yBound, zBound);

inside = this. checkifin (yBound, zBound);

if (inside)

timeGBSct=this.gbSCT (initialPosition, grainB,probGBS);

if ( $\mathrm{t}$ imeGBSct $==-1$.0)

timeElas = Copper.MAXTREL;

timeInelas $=0.0$;

\}

else

\{

timeInelas=timeGBSct;

timeElas=t_elasfirst + t_elassecond;

inside=this. checkifin (yBound, zBound);

else // 2nd elas out..

\{

Coords nTempSurfPosTwo = new Coords(this.getX(), this.getY(), this.getZ());

Coords checky = new Coords(this.getX(), this.getY(), this.getz());

noway = initialPosition.unallowedDirection (yBound, zBound);

initialPosition.moveInside (0.00001, noway)

nTempSurfPosTwo.findIntersect (initialPosition, yBound, zBound) ;

noway = nTempSurfPosTwo.unallowedDirection (yBound, zBound);

nTempSurfPosTwo.moveInside (0.00001, noway);

timeGBSct=nTempSurfPosTwo.gbSCT (initialPosition,grainB,probGBS) ; 


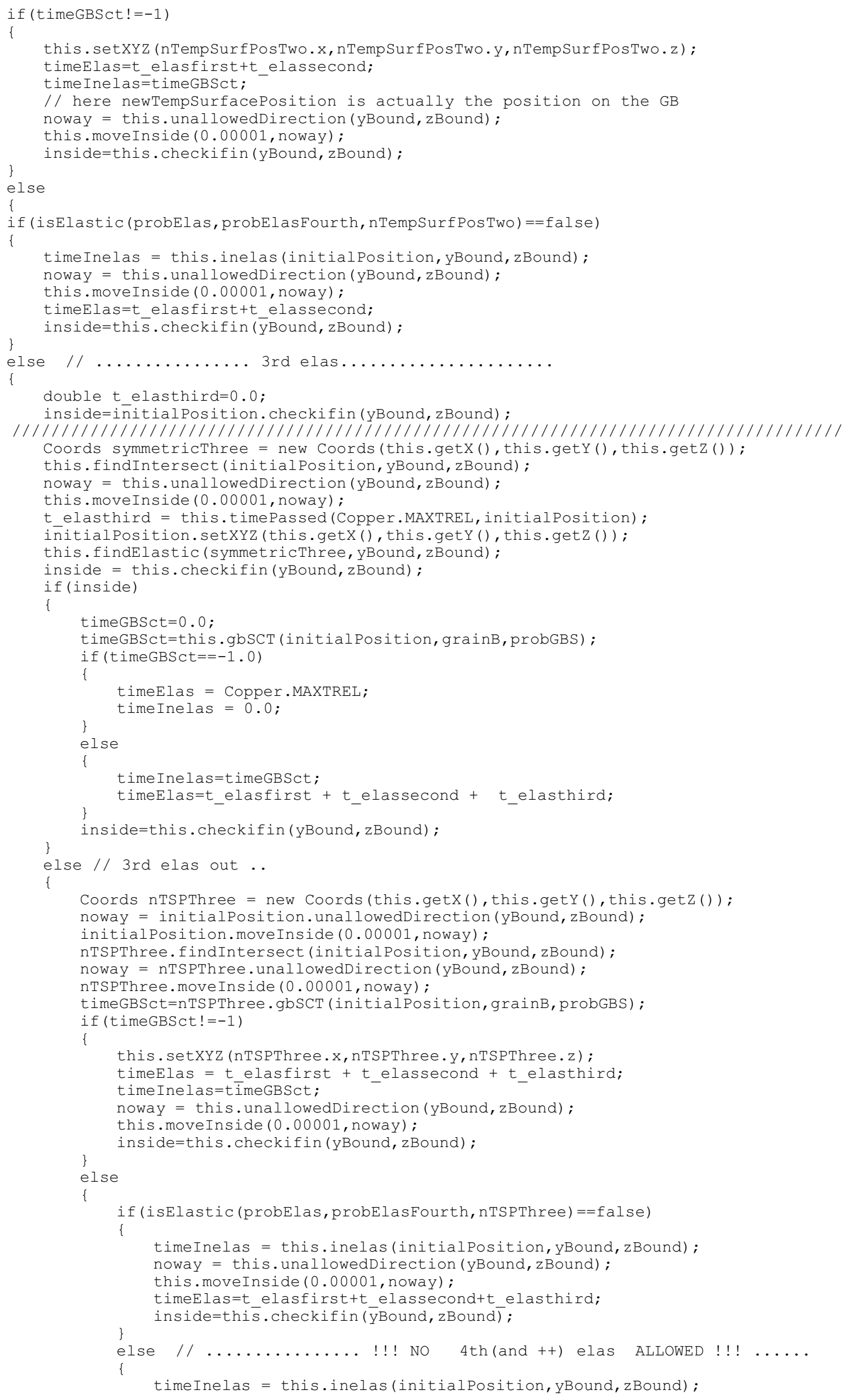




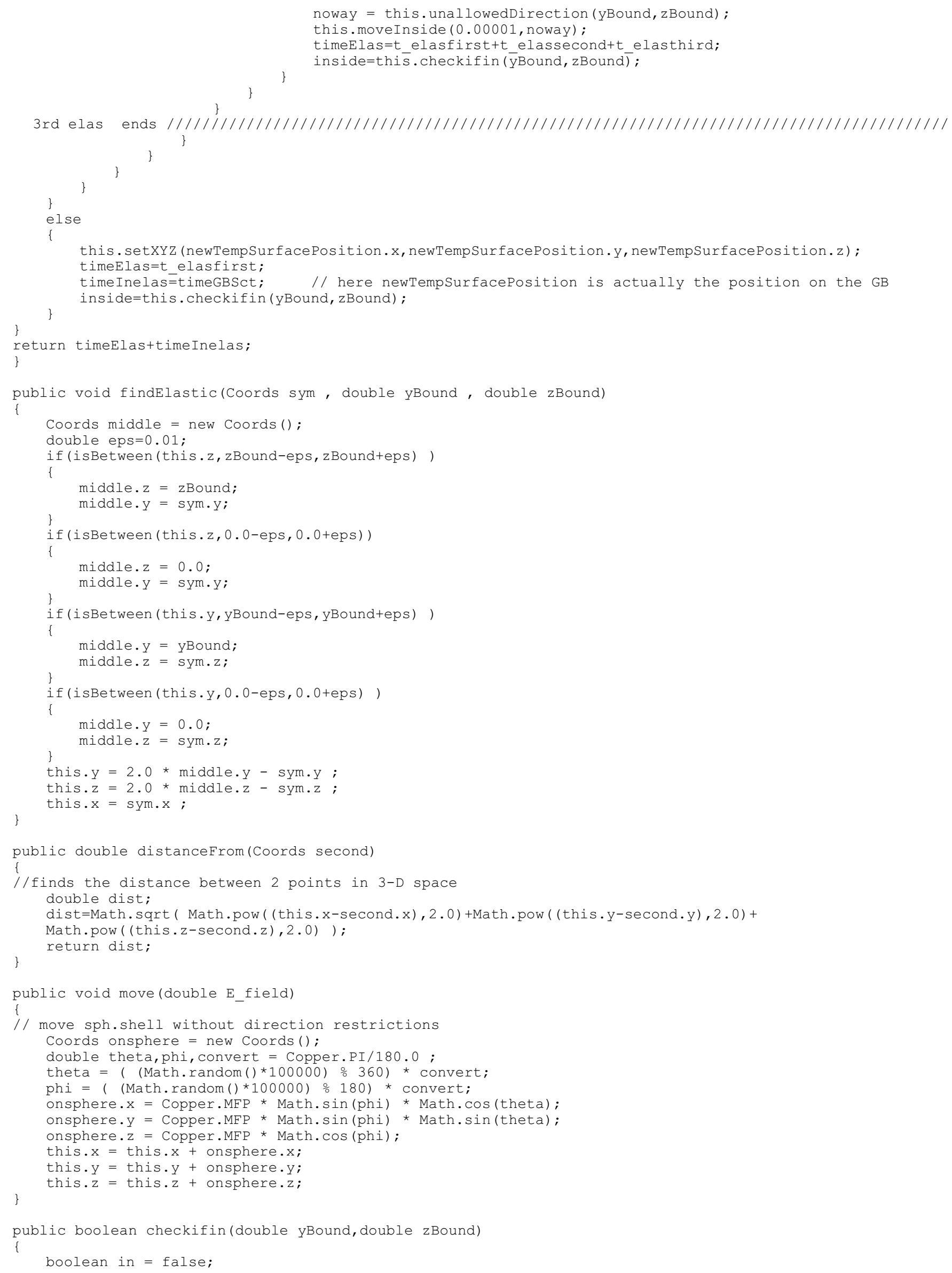




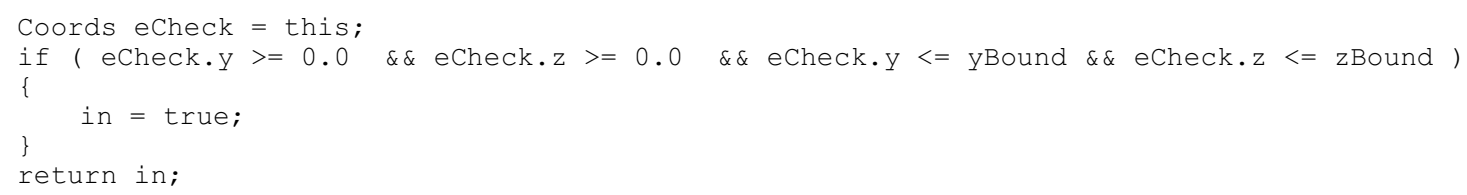




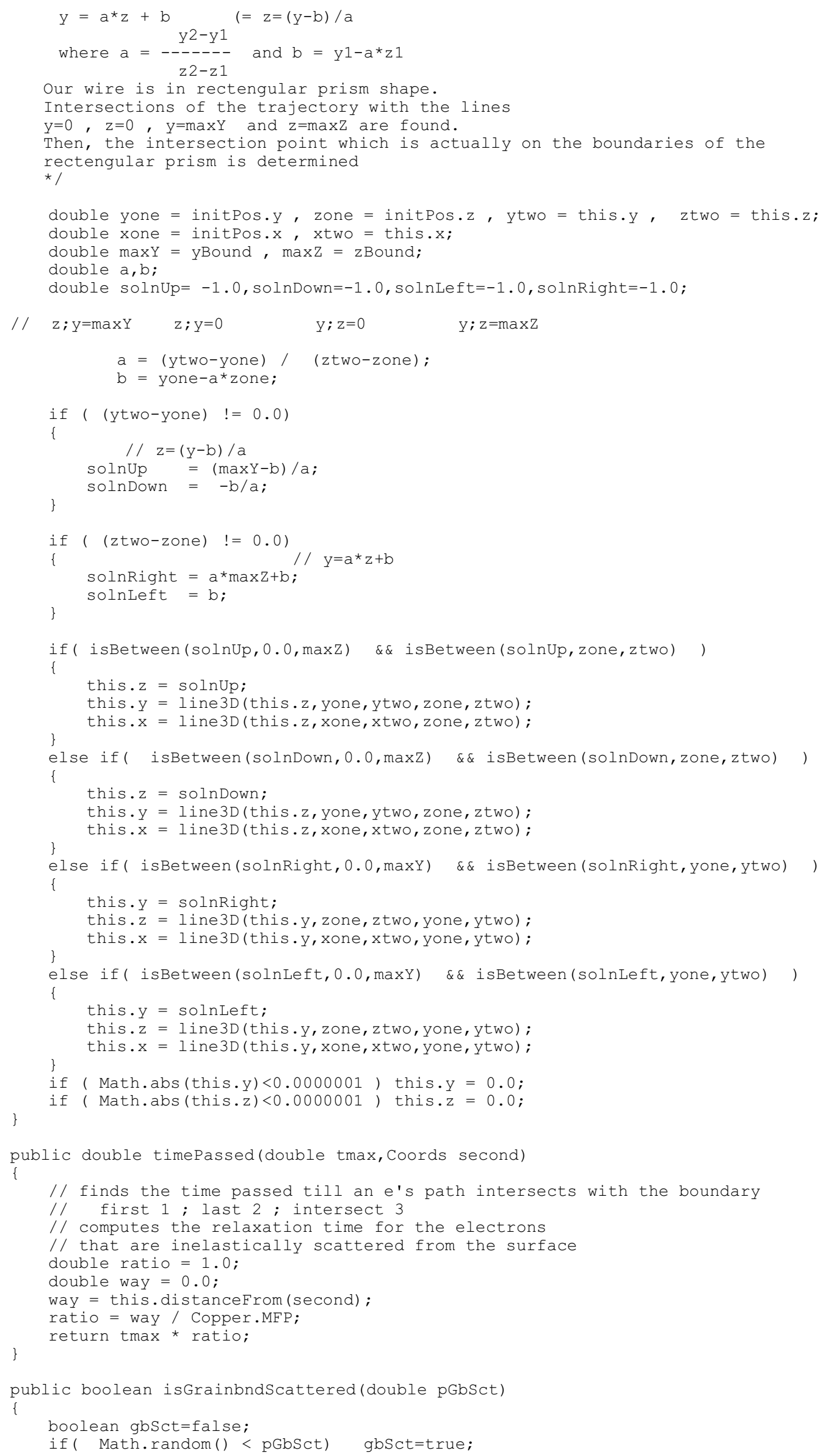




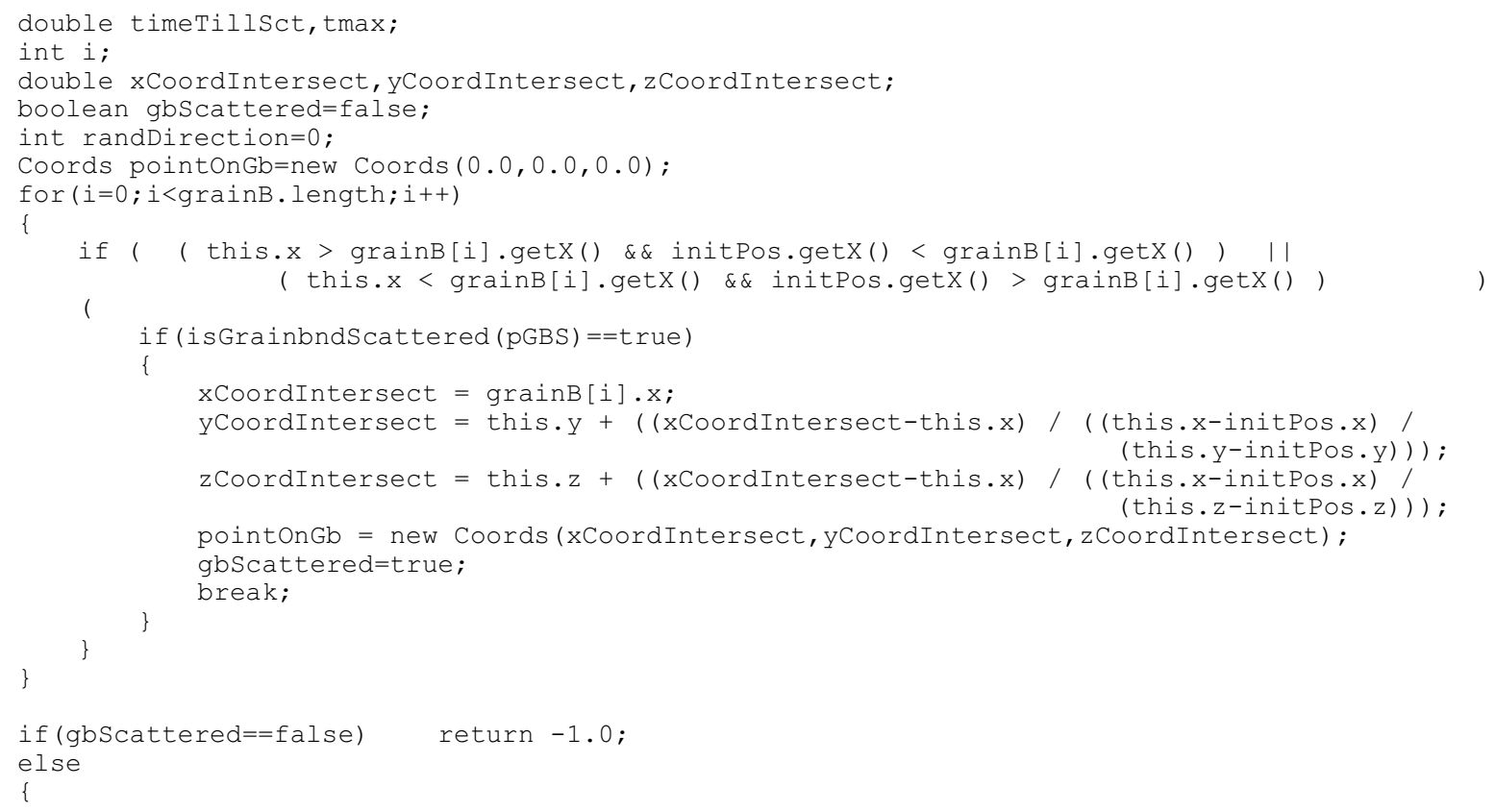




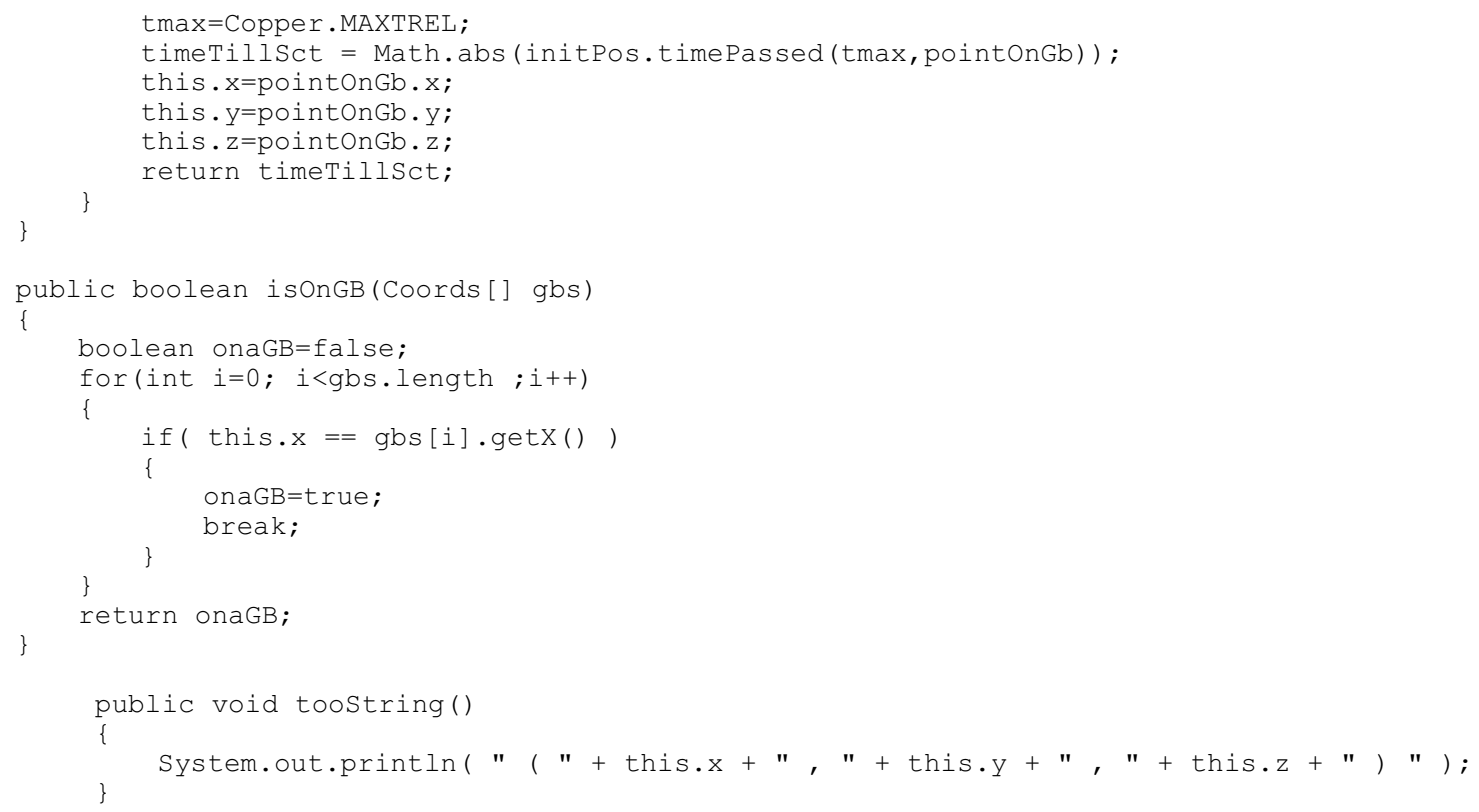

